\title{
BMJ Open Discontinuing antidepressant medication after mindfulness-based cognitive therapy: a mixed-methods study exploring predictors and outcomes of different discontinuation trajectories, and its facilitators and barriers
}

\author{
Marloes J Huijbers (D , ${ }^{1}$ Carolien Wentink, ${ }^{1}$ Esther Simons, ${ }^{2}$ Jan Spijker (D) , ${ }^{3}$ \\ Anne Speckens ${ }^{1}$
}

To cite: Huijbers MJ, Wentink C, Simons E, et al. Discontinuing antidepressant medication after mindfulness-based cognitive therapy: a mixedmethods study exploring predictors and outcomes of different discontinuation trajectories, and its facilitators and barriers. BMJ Open 2020;10:e039053. doi:10.1136/ bmjopen-2020-039053

- Prepublication history and supplementary material for this paper is available online. To view these files, please visit the journal online (http://dx.doi org/10.1136/bmjopen-2020039053).

Received 02 April 2020 Revised 15 September 2020 Accepted 30 September 2020

D) Check for updates

(C) Author(s) (or their employer(s)) 2020. Re-use permitted under CC BY-NC. No commercial re-use. See rights and permissions. Published by BMJ.

For numbered affiliations see end of article.

\section{Correspondence to}

Dr Marloes J Huijbers;

marloes.huijbers@radboudumc. $\mathrm{nl}$

\section{ABSTRACT}

Objectives This study aimed to explore predictors and outcomes associated with different trajectories of discontinuing antidepressant medication (ADM), in recurrently depressed individuals after participation in mindfulness-based cognitive therapy (MBCT). Facilitators and barriers of discontinuation were explored qualitatively. Design Mixed-methods study combining quantitative and qualitative data, drawn from a randomised controlled trial. Setting Twelve secondary and tertiary psychiatric outpatient clinics in the Netherlands.

Participants Recurrently depressed individuals $(\mathrm{N}=226)$ who had been using ADM for at least 6 months and in partial or full remission. Regardless of trial condition, we made post-hoc classifications of patients' actual discontinuation trajectories: full discontinuation $(n=82)$, partial discontinuation $(\mathrm{n}=34)$ and no discontinuation $(n=110)$ of ADM within 6 months after baseline. A subset of patients $(n=15)$ and physicians $(n=7)$ were interviewed to examine facilitators and barriers of discontinuation. Interventions All participants were offered MBCT, which consisted of eight weekly sessions in a group.

\section{Primary and secondary outcome}

measures Demographic and clinical predictors of successful discontinuation within 6 months, relapse risk within 15 months associated with different discontinuation trajectories, and barriers and facilitators of discontinuation. Results Of the 128 patients assigned to MBCT with discontinuation, only 68 (53\%) fully discontinued ADM within 6 months, and 17 (13\%) discontinued partially. Predictors of full discontinuation were female sex, being employed and lower levels of depression. Relapse risk was lower after no discontinuation (45\%) or partial discontinuation (38\%), compared with full discontinuation $(66 \%)(p=0.02)$. Facilitators and barriers of discontinuation were clustered within five themes: (1) pre-existing beliefs about depression, medication and tapering; (2) current experience with ADM; (3) life circumstances; (4) clinical support and (5) mindfulness.
Strengths and limitations of this study

- Rather than using opinions or hypothetical perspectives on tapering, this study used data on the actual discontinuation trajectories that recurrently depressed patients engaged in, and the associated predictors and outcomes.

- The facilitators and barriers reported by patients who attempted to discontinue are in accordance with and support previous findings from the qualitative literature.

- Professionals' perspectives were included to triangulate patients' perspectives on discontinuation of antidepressant medication in the qualitative study.

- Reports of relapse/recurrence may have been inflated by withdrawal or post-withdrawal symptoms, which could not be differentiated with the available data.

- Selection bias cannot be ruled out, as participation in the trial might have been influenced by perceptions and preferences regarding both mindfulness and the use of antidepressant medication.

Conclusions Discontinuing antidepressants appears to be difficult, stressing the need to support patients and physicians in this process. MBCT may offer one of these forms of support.

Trial registration number ClinicalTrials.gov Registry (NCT00928980); post-results.

\section{INTRODUCTION}

Major depressive disorder (MDD) is a highly prevalent mental disorder with a large burden of disease and high risk of recurrence. ${ }^{1}$ One of the most commonly used and effective relapse prevention strategies is maintenance 
antidepressant medication (ADM) ${ }^{2}$ Current guidelines recommend patients with MDD to continue medication for at least 2 years after remission. ${ }^{34}$ However, patients are often reluctant to use ADM for long periods because of side effects, pregnancy or interaction with other medication. As discontinuing ADM can be associated with significant withdrawal effects ${ }^{5}$ and increased relapse risk, ${ }^{6}$ there has recently been a rise in scientific and clinical interest in this area. ${ }^{78}$

One of the psychological treatment strategies that have been developed to protect against depressive relapse is mindfulness-based cognitive therapy (MBCT).${ }^{9} \mathrm{~A}$ recent meta-analysis showed that MBCT is at least as effective in reducing the risk of relapse/recurrence as ADM..$^{10}$ As MBCT provides patients support in managing uncomfortable somatic sensations and emotions, it could also help them to deal with possible withdrawal effects and increased emotional reactivity during discontinuation of ADM. ${ }^{711}$ Two randomised controlled trials (RCTs) in the UK, offering recurrently depressed patients MBCT with additional tapering support, indeed showed successful tapering of ADM in about three-quarters of their participants. ${ }^{1213}$ However, studies conducted in the Netherlands showed markedly lower levels of successful tapering, that is, about half when combined with $\mathrm{MBCT}^{6}$ or preventive cognitive therapy ${ }^{14}$ and only $6 \%$ when tapering advise was given without further support. ${ }^{15}$

The current study describes secondary analyses of an RCT, in which 249 patients with recurrent depression in remission were randomly allocated to MBCT with continued use of ADM ( $n=121)$, or to MBCT followed by discontinuation $(n=128)$. Results showed that discontinuing ADM after MBCT was associated with significantly higher relapse rates than continuing ADM after MBCT (intent-to-treat: $54 \%$ vs $39 \%$, per-protocol: $69 \%$ vs $46 \%$, respectively). ${ }^{6}$ In the original MBCT+discontinuation treatment arm, only $53 \%$ of the participants were able to fully discontinue within 6 months from baseline, $13 \%$ discontinued partially, $25 \%$ of the participants decided to continue their medication as it was despite the randomisation, and for $9 \%$ it was unknown. Notably, discontinuation also occurred in patients asked to continue their medication: $12 \%$ discontinued fully and $14 \%$ partially.

The aim of this study was to examine possible predictors and outcomes of full, partial and no discontinuation of ADM in recurrently depressed individuals who participated in MBCT in the context of the abovementioned RCT. In addition, we explored the barriers and facilitators of discontinuation by conducting in-depth qualitative interviews with a subsample of RCT participants and their attending clinicians.

\section{METHODS}

\section{Data}

Data originated from an RCT comparing MBCT followed by discontinuation or continuation of $\mathrm{ADM}^{6}{ }^{6}$ For the current paper, groups were created post hoc based on patients' actual discontinuation profiles during the study, that is, full, partial or no discontinuation of their ADM. Quantitative data were collected between September 2009 and June 2013.

We invited a purposive sample of the participants and their attending clinicians for a semistructured interview focusing on barriers and facilitators of discontinuation. These interviews were conducted after the trial (between May and August 2013) as a follow-up study specifically focusing on the barriers and facilitators of ADM discontinuation.

\section{Participants}

Patients with three or more previous depressive episodes who had been using ADM for 6 months or longer were recruited in 12 secondary and tertiary psychiatric outpatient clinics across the Netherlands between September 2009 and January 2012. For further information on the specific inclusion and exclusion criteria and process of obtaining informed consent from participants, we refer to the publication of the trial itself. ${ }^{6}$

\section{Patient and public involvement}

At the time of the study, public or patients were unfortunately not yet involved in working with the research funder to prioritise research or offering advice as members of our own project steering group. However, patients and healthcare professionals in the participating centres were regularly informed about the progress of the study via newsletters. In addition, the qualitative feedback on the barriers and facilitators of MBCT as supportive intervention during discontinuation of ADM has been directly translated into the refinement of an MBCT intervention to support ADM discontinuation, investigated in an RCT conducted between 2015 and 2019 by our group. ${ }^{16}$

\section{Procedure}

A detailed description of the study procedures in line with Consolidated Standards of Reporting Trials guidelines is provided in the RCT report. ${ }^{6}$ Eligible patients were randomly assigned to MBCT followed by guided discontinuation of ADM or to MBCT with continuation of ADM. Follow-up quantitative assessments (measures of depression, relapse/recurrence, ADM usage) took place $3,6,9,12$ and 15 months after baseline.

For the qualitative part of the study, we adhered as much as possible to the Standards for Reporting Qualitative Research. ${ }^{17}$ A subset of 15 participants from the MBCT+discontinuation group were purposively sampled on the basis of age, sex and discontinuation profile (ie, fully, partially or not discontinued). We kept inviting participants until saturation of the data was established. For the purpose of triangulation, we also included physicians who had guided discontinuation. Interviews were semi-structured, individual and by telephone. Patients and attending physicians were asked the following questions: (1) 'How did the tapering go?', (2) 'What expectations did you have about tapering ADM?', (3) 'What 
hampered your (the patients') discontinuation process?', (4) 'What facilitated your (the patients') discontinuation process?'; (5) 'What was the role of mindfulness in your (the patients') process of discontinuation?' and (6) 'Do you have any suggestions for future guidance on tapering ADM?'. The interviews were conducted by CW and ES, both women. CW was a graduate student in Psychology during the interviews and data analysis, and a psychologist and $\mathrm{PhD}$ student researching $\mathrm{ADM}$ discontinuation in primary care at the time of writing. ES was a medical student completing her research internship. Neither of them were acquainted with the participants prior to the interviews.

\section{Interventions}

Mindfulness-based cognitive therapy

MBCT was largely based on the protocol by Segal and Teasdale $^{9}$ and consisted of eightweekly sessions of 2.5 hours and 1 day of silent practice between the sixth and seventh session. It was delivered in groups of 8-12 participants and included mindfulness meditation practices, group inquiry, cognitive behavioural elements, interactive psycho-education and home practice.

\section{Discontinuation or continuation of ADM}

Patients in the discontinuation arm were asked and recommended to gradually withdraw from their ADM over a period of 5 weeks, starting after the seventh session of MBCT, with specified steps for each of the commonly used types of antidepressants ${ }^{18}$ and supervised by their attending physician (mostly psychiatrists) in 3-12 consultations. Patients in the continuation arm were offered a minimum of one consultation. Psychiatrists were instructed to maintain or reinstate an adequate dose of $\mathrm{ADM}$, and recommendations to manage side effects were provided. Full discontinuation was defined as tapering to nil milligrams within 6 months after baseline. Partial discontinuation was defined as tapering to a lower dose and 'no discontinuation' was defined as maintaining or increasing the initial therapeutic dose of ADM throughout the first 6 months after baseline.

\section{Outcome measures}

Relapse/recurrence rates were compared between the three discontinuation subgroups (full, partial, not). It was assessed using the Structured Clinical Interview (SCID) for Diagnostic and Statistical Manual of Mental Disorders-4th Edition (DSM-IV) ${ }^{19}$ by trained research assistants every 3 months during the 15-month follow-up period. The inter-rater reliability between first and second (blind) ratings was found to be substantial (kappa $=0.70, \mathrm{p}<0.001$, 95\% CI=0.456-0.942). Baseline severity of depressive symptoms was measured with the Inventory for Depressive Symptomatology-clinician rated. ${ }^{20}$

\section{Statistical and qualitative analysis Quantitative analysis}

All analyses were performed using SPSS Statistics V.20.0. Probability values lower than 0.05 (two-tailed) were considered significant for all analyses. Possible differences in baseline characteristics between participants who discontinued fully, partially or not at all were examined using independent sample t-tests for continuous and Pearson $\chi^{2}$ tests for categorical variables.

Relapse/recurrence rates across the entire trial sample, differentiated into three discontinuation profile groups (full, partial, not) were compared with a Pearson $\chi^{2}$ test and differences in time to relapse/recurrence were analysed using a Cox regression proportional hazards model. Baseline depression severity and number of previous episodes (log transformed) were included as covariates because these factors have been consistently associated with an increased relapse risk. ${ }^{21}$ Patients whose follow-up data were unavailable or who did not experience a relapse/recurrence before the end of the follow-up period were treated as censored observations.

\section{Qualitative analysis}

The qualitative interviews were audio-taped, transcribed verbatim and imported in the scientific qualitative research software program ATLAS.ti (V.7). ${ }^{22}$ We used a thematic approach to analyse the data, with a focus on barriers and facilitators of the discontinuation process, including previous expectations and the possible role of mindfulness in that process. The underlying framework for the study can be described as a combination of a phenomenological and praxis-oriented approach, as we were both interested in participants' views and in behavioural strategies that helped or hindered discontinuation. Analysis started as soon as the first data were collected and continued with each additional interview. Two researchers (CW, ES) coded the transcripts independently to minimise subjectivity. Subsequently codes were modified and categorised as various facilitators and barriers by the full research team, also consisting of a professor of psychiatry and mindfulness teacher with prior experience with qualitative research (AS) and a psychologist and post-doc researcher who also worked as a mindfulness teacher $(\mathrm{MJH})$. The cycle of comparison and reflection on 'old' and 'new' themes was repeated several times. Eventually, characteristic quotes were used to illustrate the final themes and subthemes. The original Dutch quotes in this article were translated into English by the authors.

\section{RESULTS}

\section{Quantitative results}

Flow of ADM discontinuation and intervention adherence

The flow of participants and their ADM use is shown in figure 1. Of the 249 participants randomised, 128 were allocated to MBCT+discontinuation and 121 to MBCT+ADM. From 23/249 (9\%) patients, we had insufficient information about ADM use due to early dropout. Consequently, descriptive and statistical analyses were performed on 226 participants. 


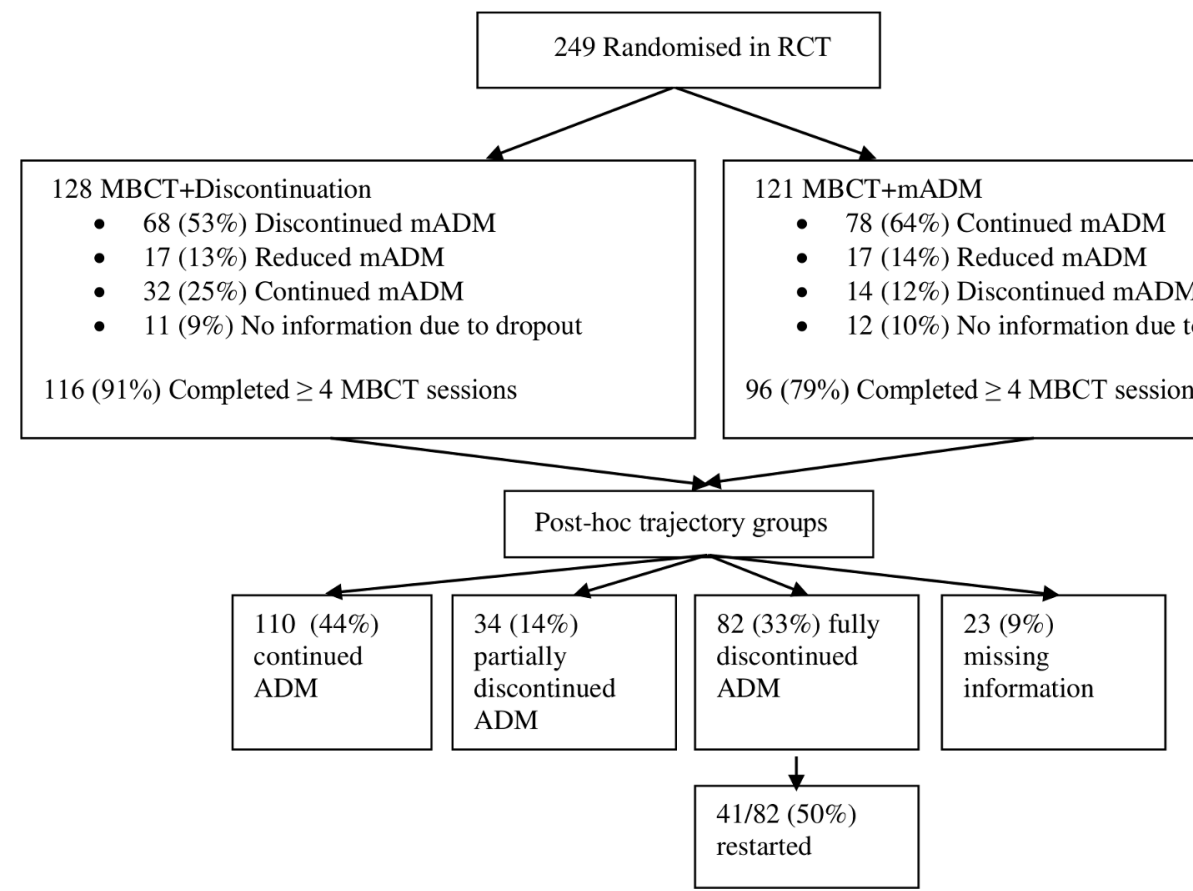

Figure 1 Flow chart of participants, their adherence to mindfulness-based cognitive therapy (MBCT) and their use of antidepressant medication (ADM). mADM, maintenance ADM; RCT, randomised controlled trial.

Of the 82 patients who fully discontinued ADM within 6 months after baseline, 41 (50\%) restarted ADM at some point during the 15-month study period. Based on the SCID interviews, this might have been related to a relapse in $31 / 41(76 \%)$ of them.

Adherence to MBCT sessions differed significantly between those with a full discontinuation profile $\mathrm{M}=7.2 \pm 1.5$ versus the no discontinuation group, $\mathrm{M}=6.4 \pm 2.0(\mathrm{p}=0.003)$. Those who partially discontinued were in between, with an attendance of $M=7.0 \pm 1$.4. The number of medication consultations also differed significantly among the groups, being highest for those with a full discontinuation profile $(\mathrm{M}=3.0 \pm 2.0$; range $0-13)$ versus partial discontinuation ( $\mathrm{M}=2.3 \pm 1.4$; range $1-6)$ $(\mathrm{p}=0.03)$ versus no discontinuation group $(\mathrm{M}=1.6 \pm 1.5$; range $0-11)(\mathrm{p}<0.00)$. The partial and full discontinuation group did not differ significantly $(\mathrm{p}=0.06)$.

\section{Demographic and clinical characteristics}

The demographic and clinical characteristics for the different profile groups are summarised in table 1. In comparison with those who did not discontinue, women were more likely to fully discontinue than men $(76 \%$ vs 53\%; $\mathrm{p}=0.026$ ) and employed participants more likely than those who were unemployed ( $78 \%$ vs $56 \%$; $\mathrm{p}=0.002$ ). Those who fully discontinued also had significantly less depressive symptoms at baseline than those who did not ( $\mathrm{M}=10.9$ (SD 8.8) vs $\mathrm{M}=14.4$ (SD 10.6), $\mathrm{p}=0.018 ; \mathrm{d}=0.37$ ).

\section{Relapse and recurrence}

Rates of relapse were $66 \%$ for full discontinuation, $38 \%$ for partial discontinuation and $45 \%$ for no discontinuation. As illustrated in figure 2, Cox regression analysis with baseline level of depression and number of previous episodes (log transformed) as covariates showed that there were significant differences in terms of risk of relapse across the three groups $(\mathrm{p}=0.02)$. Compared with the full discontinuation group, those who did not discontinue had a lower risk of relapse $(\mathrm{HR}=0.67 ; 95 \% \mathrm{CI}=0.45-$ $0.99 ; \mathrm{p}=0.04$ ). The lowest risk of relapse was seen for those who partially discontinued, compared with full discontinuation $(\mathrm{HR}=0.47 ; 95 \% \mathrm{CI}=0.26-0.87 ; \mathrm{p}=0.02)$. There was no significant difference in relapse risk between partial and no discontinuation $(\mathrm{p}=0.27)$.

\section{Qualitative results}

The interviewed patients consisted of eight women and nine men, with a mean IDS-score at baseline of $11 \pm 7.8$. See table 2 for their characteristics. Nine patients discontinued their ADM fully, three partially and three did not discontinue their ADM. The time elapsed between the final trial assessment and the qualitative interview was 0-23 months $(\mathrm{Mn}=11$ months \pm 6 ).

Five themes emerged from the patient interviews: (1) patients' pre-existing beliefs about depression, medication and tapering; (2) current experience with antidepressants; (3) psychosocial conditions and physical problems; (4) clinical support and (5) participating in MBCT. The most significant subthemes are described in more detail below. See online supplemental material 1 for an overview of themes, subthemes and illustrating quotes.

Pre-existing beliefs about depression, medication and tapering Facilitators

Being aware that others are also subject to periods of low mood and feeling downhearted occasionally seemed to facilitate the process of discontinuation. This was accompanied by the realisation that medication is not always 
Table 1 Baseline demographic and clinical characteristics of 226 patients with recurrent depression receiving mindfulnessbased cognitive therapy who subsequently engaged in full, partial or no discontinuation of maintenance antidepressant medication (mADM) (adapted from Huijbers et al, 2016)

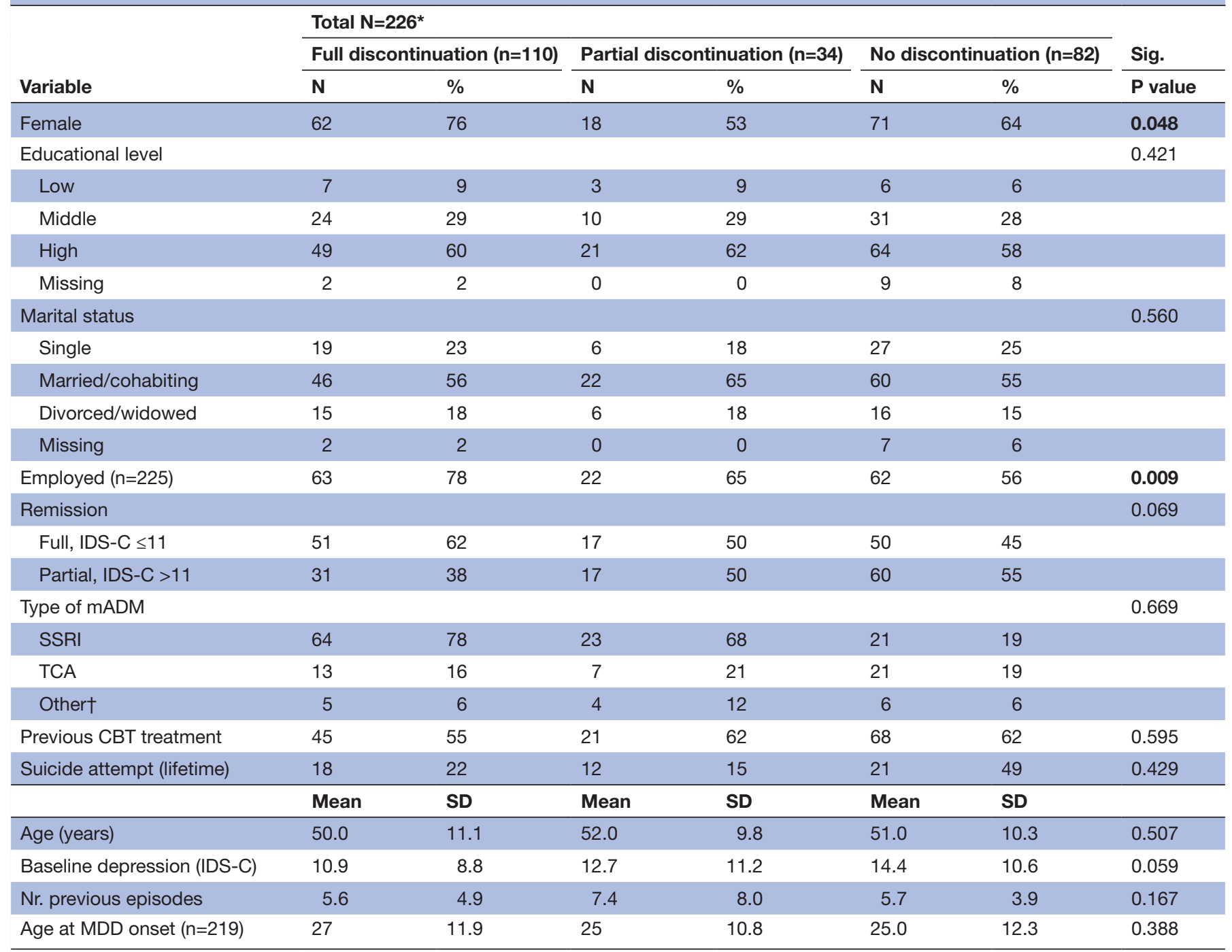

*Excluding 23 of the original 249 trial participants due to missing data regarding discontinuation. †Including serotonin-norepinephrine reuptake inhibitors, monoamine oxidase-inhibitors and mirtazapine.

CBT, cognitive behavioural therapy; IDS-C, Inventory of Depressive Symptomatology-clinician rated; MDD, major depressive disorder; SSRI, selective serotonin reuptake inhibitor; TCA, tricyclic antidepressant.

needed to suppress those feelings. Although most respondents had regarded their medication as helpful during their depression, some patients considered taking pills for long periods of time as unhealthy and even 'harmful'. Respondents also talked about their wish to manage without pills.

\section{Barriers}

Several respondents mentioned that they were (told to be) missing a specific substance in their brain and therefore needed ADM. They considered their condition as chronic and medication as necessary. Not surprisingly, these people were reluctant to (fully) taper their medication. Another central theme was the fear of relapse in depression. Several patients expressed concern that this would happen. On a related note, many participants had tried to come off medication in the past and experienced difficulties, that is, withdrawal symptoms.

\section{Current experience with antidepressants}

\section{Facilitators}

There were patients describing uncertainty about the benefits of taking their medication. In addition, the occurrence of unwanted or 'side' effects emerged as a theme. In addition, being able to adopt a personalised tapering schedule facilitated the discontinuation process. Such a personalised schedule typically contained adaptations with regard to the duration and magnitude of the dose reductions, or were much more flexible so that tapering could be guided by their own mental health state. 

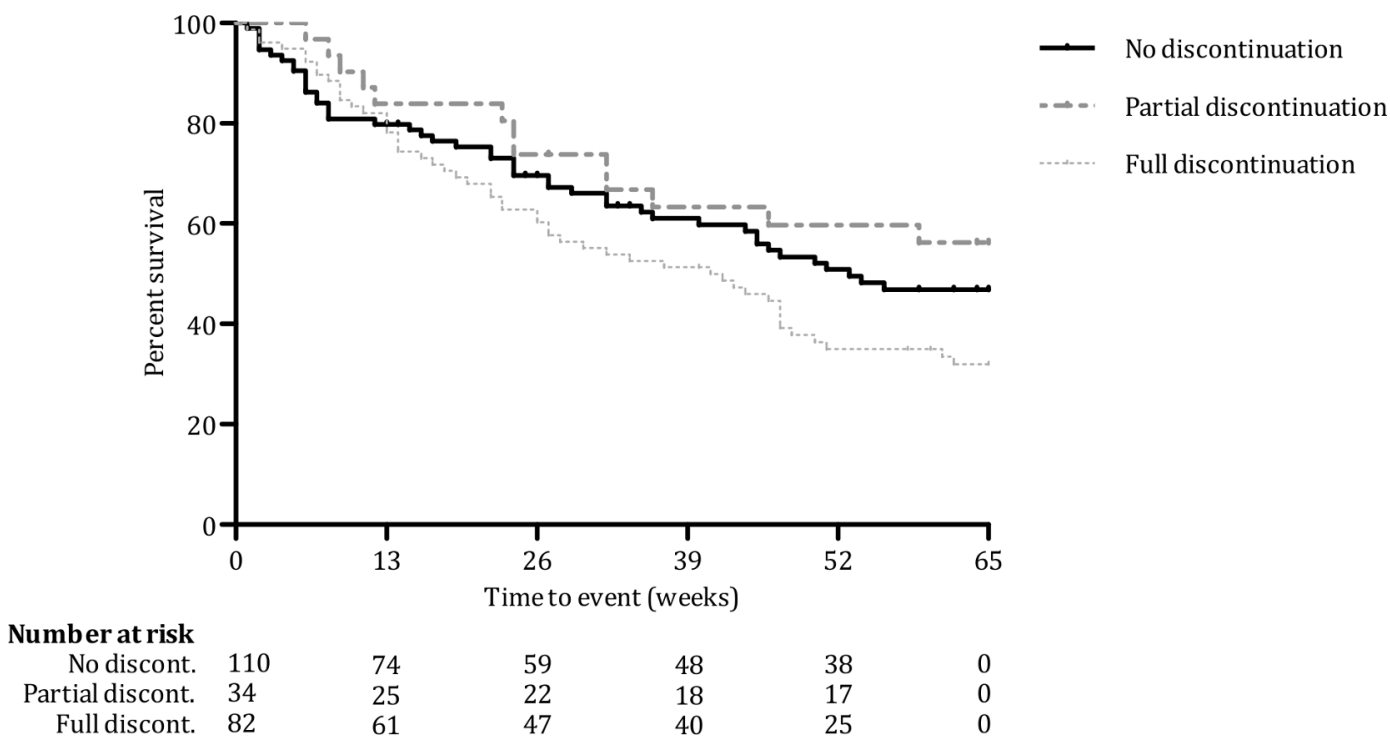

$\begin{array}{lllll}74 & 59 & 48 & 38 & 0 \\ 25 & 22 & 18 & 17 & 0 \\ 61 & 47 & 40 & 25 & 0\end{array}$

Figure 2 Survival curves over 15-month follow-up for risk of relapse in recurrently depressed patients with different profiles of discontinuing antidepressant medication: fully $(n=82)$, partially $(n=34)$ or not $(n=110)$.

\section{Barriers}

Some participants for whom ADM were still beneficial in managing depressive symptoms did not start discontinuation. Withdrawal effects appeared one of the major reasons to interrupt discontinuation, restart or increase the dose of medication. For many patients the tapering speed according to the RCT guideline was considered too fast. One patient said, for example: "I was using Citalopram and I believe I had to taper within 2 weeks, but I was suffering quite a lot from withdrawal symptoms, so therefore, after consultation, tapered somewhat more slowly."

\section{Life circumstances}

\section{Facilitators}

In a relatively quiet period, people appeared more likely to start and proceed with tapering.

\section{Barriers}

If there were stressful circumstances (for example, workrelated problems), a lack of support from family or friends, or health problems, people seemed more reluctant to start or continue the tapering process.

\begin{tabular}{|c|c|c|c|c|c|}
\hline Patient & Sex & Age range & IDS at baseline & $\begin{array}{l}\text { Discontinuation } \\
\leq 6 \text { months }\end{array}$ & $\begin{array}{l}\text { Relapse } \\
\leq 15 \text { months }\end{array}$ \\
\hline $\mathrm{P} 1$ & Male & $30-39$ & 9 & Partially & No \\
\hline $\mathrm{P} 2$ & Male & $40-49$ & 15 & Not & No \\
\hline P3 & Female & $30-39$ & 10 & Fully & Yes \\
\hline $\mathrm{P} 4$ & Female & $20-29$ & 10 & Fully & Yes \\
\hline P5 & Male & $60-69$ & 0 & Fully & Yes \\
\hline P6 & Female & $50-59$ & 13 & Partially & Yes \\
\hline P7 & Male & 70-79 & 10 & Fully & No \\
\hline P8 & Female & $50-59$ & 17 & Partially & Yes \\
\hline P9 & Male & $50-59$ & 3 & Not & Yes \\
\hline P10 & Male & $60-69$ & 30 & Fully & Yes \\
\hline P11 & Female & $60-69$ & 20 & Not & Yes \\
\hline P12 & Female & $50-59$ & 7 & Fully & Yes \\
\hline P13 & Male & $50-59$ & 14 & Fully & No \\
\hline P14 & Female & $30-39$ & 14 & Fully & Yes \\
\hline P15 & Female & $60-69$ & 0 & Fully & No \\
\hline
\end{tabular}

IDS, Inventory of Depressive Symptomatology. 
Clinical support

Facilitators

Availability and accessibility of professional guidance was an important facilitator, both before and during the tapering process. Patients mentioned the importance of bringing in their own ideas of how clinical support would ideally look like, and having the freedom to choose for themselves what they felt was best. Reassurance about being able to restart medication in case of deterioration was also described as helpful.

\section{Barriers}

In some cases the attending clinician would advise against discontinuation, which was mentioned as a barrier: "He [the psychiatrist] said: "I would not do it with your history and family matters". But I wanted to taper (...) and so I did." Another barrier that emerged from the interviews was the impression that, possibly related to the context of the RCT, tapering was mandatory.

\section{Mindfulness practice \\ Facilitators}

Participants mentioned peer support in MBCT as a facilitating factor. Witnessing how other people deal with similar problems and sharing fears and insecurities about tapering were regarded as helpful and reassuring. In addition, mindfulness itself had been a source of support during tapering, for example being able to distinguish feelings of stress from a depressive relapse and recognising that these were triggered by difficult psychosocial circumstances, which did not necessarily require to restart medication. Mindfulness practice also provided an alternative method to prevent depressive relapse, by recognising periods of increased vulnerability and using other approaches rather than increasing the dose of ADM (eg, daily walks in nature, seeing friends and reducing workload).

\section{Barriers}

The MBCT group context could also negatively impact the process. MBCT groups included participants from both arms of the RCT, including those who were asked to continue ADM after MBCT. It was mentioned that sometimes fellow group members, who continued their medication, advised against discontinuation.
Facilitators and barriers as reported by attending clinicians

Fourteen patients gave permission to interview their attending physician, of whom seven were willing and able to participate. See table 3 for their characteristics.

Professionals' perspectives generally showed a large overlap with the views expressed by participants. For example, the benefits of tapering slowly and with a personalised tapering scheme clearly emerged from these interviews. In terms of barriers, the pivotal roles of negative experiences with tapering in the past, worrying about symptoms and possible relapse, and stressful circumstances were mentioned.

Some different themes emerged from these interviews as well. Clinicians reported feeling reluctant to discontinue ADM because of their own worries about patients having a relapse, especially in case of a long psychiatric history or comorbidity. They spoke more specifically about ADM characteristics (half-life time) and switching to a different type of ADM before fully discontinuing as a possible facilitator. They also mentioned the possible use of other psychological interventions to help patients cope with emerging symptoms. Regarding barriers, clinicians were particularly concerned about nocebo effects when discussing potential withdrawal effects, suggesting to provide some information but avoiding being very specific about it.

\section{DISCUSSION}

\section{Principal findings}

The current paper provides quantitative post-hoc data from an RCT, describing the flow, characteristics and outcomes of patients with recurrent depression who discontinued ADM fully, partially or not at all. Quantitative data were complemented by qualitative data on the barriers and facilitators of ADM withdrawal. Remarkably, there were relatively low rates of adherence to the ADM protocol, including crossover. These non-compliance rates and apparent difficulties with discontinuation are in line with previously published studies on (preventive) cognitive therapy for recurrently depressed patients ${ }^{14}$ and anxiety disorders. $^{23}$

However, two UK studies with an MBCT+discontinuation $\operatorname{arm}^{12}{ }^{13}$ found much better discontinuation and

\begin{tabular}{lllll}
\hline \multicolumn{2}{l}{ Table 3} & Professionals' characteristics & & \\
Professional & Sex & Age range & Function & Institute \\
\hline PF1 & Female & $50-59$ & Psychiatrist & University medical centre \\
PF2 & Female & $30-39$ & Psychiatrist in training & University medical centre \\
PF3 & Female & $40-49$ & Psychiatrist & Mental health institute \\
PF4 & Female & $40-49$ & Physician & University medical centre \\
PF5 & Male & $60-69$ & Psychiatrist & Private practice \\
PF6 & Female & $50-59$ & Psychiatrist & University medical centre \\
PF7 & Male & $40-49$ & Psychiatrist & Mental health institute \\
\hline
\end{tabular}


relapse rates. Some possible explanations for these differences deserve attention. First, the UK studies were conducted in a primary care setting and ours in secondary care. Discontinuation of ADM in secondary care may be more challenging due to longer and more complicated psychiatric, and possibly medical, histories. In addition, the role of healthcare professionals might be different: general practitioners (GPs) who guided discontinuation in the UK may have offered their patients a more psychologically oriented framework for understanding depression whereas psychiatrists in the Netherlands may have offered a more biologically oriented one. In fact, some of them appeared to have actively advised against discontinuation. In our trial patients participated in mixed MBCT groups also including patients continuing their ADM, so guided tapering took place outside the MBCT context. In the UK-based studies, MBCT groups were homogeneous, allowing the tapering process to be a more integrated part of the training.

It is important to keep in mind that the analyses of the current study are not based on the original intervention and control conditions, but on the actual ADM continuation or discontinuation in the entire study population. In terms of predictors, full discontinuation occurred more frequently in women, and in those who were employed. Indeed, the qualitative data point to psychosocial stressors as possible barriers to discontinuation, and problems with finding or holding on to a suitable job might be one of them. Residual symptoms of depression may be a predictor of discontinuation too: levels of baseline depression were lower in the full versus no discontinuation group. In contrast, the qualitative data suggest that if ADM are considered an effective treatment to reduce or manage depressive symptoms, patients are less likely to taper, and vice versa. Possibly, these beliefs may vary over time and across circumstances. For example, a patient who considers tapering because ADM has not been very effective, but would nevertheless postpone this because of a current episode of depression. Aside from baseline depression levels, post-hoc analyses by Kuyken $e t$ $a l^{12}$ suggested that patients with a more severe psychiatric history (earlier age of onset and greater severity of the last episode) were more likely to taper their ADM after MBCT. In the current study, age at onset was no predictor of successful discontinuation, neither was the number of previous episodes. The relation between clinical characteristics and discontinuation appears to be a complex interplay between several other factors. ${ }^{24}$

Even in those participants who discontinued completely in the current study, more than half restarted medication within the next 9 months, possibly related to relapse or recurrence of depression. Relapse rates were indeed substantially higher for fully discontinued patients than for partially and not discontinued. These differences seem clinically relevant, and may even advocate partial rather than full discontinuation. While linear tapering regimes are commonplace, the most challenging part of the withdrawal process may occur at the lowest doses.
As this might have to do with hyperbolic dose-response relationships between drugs such as selective serotonin reuptake inhibitors (SSRIs) and their receptor, ${ }^{25}$ it has been suggested that 'stop slow if you go low' regimes may help to minimise withdrawal symptoms. ${ }^{26}$ Although the use of 'tapering strips' can be a suitable way to taper gradually, ${ }^{27}$ for many types of ADM these are not yet available.

In line with a recent systematic review, ${ }^{24}$ worry and fear of relapse emerged as clear barriers to discontinuation in the qualitative interviews. These fears appear to exist not only for patients, but also for attending physicians. Some professionals reported being anxious about responsibility for deterioration and sometimes feeling unable to help, which has also been reported in previous studies. ${ }^{28}$ For both patients and professionals, accessibility and availability of support during tapering is important.

\section{Strengths and weaknesses}

A major strength of the current study is that we combined quantitative and qualitative data to investigate what makes it more difficult or easy to discontinue ADM. Rather than using opinions or hypothetical perspectives on tapering, we report data on the actual tapering process in an RCT, including clinical outcomes, in which full, partial and no discontinuation were defined before the study started. ${ }^{18}$ In addition, we looked at professionals' perspectives to triangulate patients' perspectives on discontinuation of $\mathrm{ADM}$ in the qualitative study.

One of the limitations of the current study is that reports of relapse/recurrence may have been inflated by withdrawal or post-withdrawal symptoms. Chouinard and Chouinard have developed criteria permitting identification of three types of withdrawal problems associated with SSRIs: new withdrawal symptoms, rebound and persistent post-withdrawal disorder, which can be differentiated from relapse and recurrence. ${ }^{29}$ As withdrawal symptoms were not included as an outcome measure, we could unfortunately not differentiate this in our RCT.

Another limitation is that there is no control group of patients withdrawing from ADM without MBCT. Consequently, predictors of discontinuation of antidepressants with MCBT might be predictors of the take up of MCBT rather than discontinuation of ADM. The same issue might apply to the discontinuation outcomes. These issues might be conflated by the differences found in attendance at MCBT sessions. Although we did collect some baseline demographic and clinical characteristics that might influence discontinuation, this obviously does not include all the factors that a prescribing clinician would consider before recommending discontinuation. For instance there are no measures of patient's subjective readiness to discontinue, mental and physical comorbidity or other baseline medication which might lead to adverse effects on the patient. This is a limitation of predictors of discontinuation and may confound those factors that have been identified as well as outcome. These factors may also be important to understand why there might be 
differences in outcome with MCBT on ADM discontinuation across studies.

In addition, we cannot rule out selection bias, as participation in the trial might have been influenced by perceptions of both mindfulness and ADM. Participants in the qualitative part of the study may be more positive about MBCT than those who dropped out of the intervention. In addition, given the delay between ADM discontinuation and the qualitative interview, memory bias may have occurred with regard to its barriers and facilitators. In addition, although we specifically asked about patients' experiences within the trial, their responses appeared to also include previous and later experiences. Moreover, the number of professionals contributing to the qualitative interview data was rather small.

\section{Clinical recommendations}

First and foremost, our findings clearly point to the necessity of up-to-date, accessible and professional guidance for those who wish to come off their ADM. A recently published shared decision-making tool ${ }^{30}$ might assist patients and their clinicians in their consultations. Second, a personalised tapering approach seems essential to enable successful tapering. With accumulating evidence suggesting that slow tapering is associated with better outcomes,${ }^{31}$ it is important to slow down the pace of tapering on the basis of patients' preferences and needs. Finally, results from the current study point to the possible clinical relevance of tapering to low doses of ADM rather than complete withdrawal. This might prevent withdrawal symptoms, whether neurochemical or psychological, empower patients by letting them choose their optimal dose, and reduce side effects and healthcare costs.

\section{Research implications}

So far, it remains unclear whether the increased risk of relapse and withdrawal symptoms are a direct effect of neurobiological changes, or an indirect effect driven by psychological mechanisms such as fear of relapse, negative expectations based on previous failed tapering attempts or nocebo effects caused by information about withdrawal symptoms. To disentangle these effects at a more fundamental level, a double-blind withdrawal study with active versus placebo pills should be conducted.

In addition, future research might focus on the effectiveness of protocolised tapering support interventions and existing psychological interventions that might be helpful to manage withdrawal symptoms and depression. Besides MBCT, preventive cognitive therapy might be a valuable option. ${ }^{14}$ Future studies should include homogeneous groups of patients who are all in the same phase of discontinuation. We are currently conducting an RCT in primary care inviting long-term ADM users who have made a shared decision to discontinue, are supported by mental health assistants or their GP in devising and monitoring their tapering process, and are either offered additional MBCT or not. ${ }^{16}$

\section{Author affiliations}

${ }^{1}$ Psychiatry, Radboudumc, Nijmegen, Gelderland, The Netherlands ${ }^{2}$ Primary and Community Care, Radboudumc, Nijmegen, The Netherlands ${ }^{3}$ Expertise Centre for Depression, Pro Persona Locatie Tarweweg, Nijmegen, Gelderland, The Netherlands

Acknowledgements The authors would like to acknowledge all participants who have contributed to this work, as well as all health care professionals who have participated in the trial and those who contributed to the qualitative interviews.

Contributors MJH and AS led the RCT from which the quantitative data were drawn. For the current study, MJH,CW, JS and AS formulated the study design. $\mathrm{MH}$ and CW collected data. ES assisted with data collection. MJH and CW contributed to data analysis and data interpretation. MJH wrote the manuscript and prepared the figures and tables. CW, ES, JS and AS edited the manuscript.

Funding The RCT from which data were drawn was funded by ZonMW, the Netherlands Organization for Health Research and Development (Grant no. 170992903 awarded to Prof. A.E.M. Speckens). No additional funding was requested for the current mixed-methods study.

Disclaimer The funder had no role in the writing of the manuscript or the decision to submit it for publication.

Competing interests $\mathrm{MH}$ and AS report grants from ZonMW Doelmatigheid during the conduct of the study.

Patient consent for publication Not required.

Ethics approval The study was approved by the Medical Ethics Committee Arnhem-Nijmegen (nr. 2008/242).

Provenance and peer review Not commissioned; externally peer reviewed.

Data availability statement Data are available upon reasonable request. We aim to make our data available for other researchers as much as possible, although with a restricted access policy. As data are currently not yet filed in a public repository, researchers interested in reusing our data are invited to contact the authors.

Supplemental material This content has been supplied by the author(s). It has not been vetted by BMJ Publishing Group Limited (BMJ) and may not have been peer-reviewed. Any opinions or recommendations discussed are solely those of the author(s) and are not endorsed by BMJ. BMJ disclaims all liability and responsibility arising from any reliance placed on the content. Where the content includes any translated material, BMJ does not warrant the accuracy and reliability of the translations (including but not limited to local regulations, clinical guidelines, terminology, drug names and drug dosages), and is not responsible for any error and/or omissions arising from translation and adaptation or otherwise.

Open access This is an open access article distributed in accordance with the Creative Commons Attribution Non Commercial (CC BY-NC 4.0) license, which permits others to distribute, remix, adapt, build upon this work non-commercially, and license their derivative works on different terms, provided the original work is properly cited, appropriate credit is given, any changes made indicated, and the use is non-commercial. See: http://creativecommons.org/licenses/by-nc/4.0/.

\section{ORCID iDs}

Marloes J Huijbers http://orcid.org/0000-0002-3381-9691

Jan Spijker http://orcid.org/0000-0003-1480-9647

\section{REFERENCES}

1 Richards D. Prevalence and clinical course of depression: a review. Clin Psychol Rev 2011;31:1117-25.

2 Gueorguieva R, Chekroud AM, Krystal JH. Trajectories of relapse in randomised, placebo-controlled trials of treatment discontinuation in major depressive disorder: an individual patient-level data metaanalysis. Lancet Psychiatry 2017;4:230-7.

3 American Psychiatric Association. Practice guideline for the treatment of patients with major depressive disorder. 3rd ed. APA, 2010.

4 National Institute for Health and Care Excellence. Depression in adults: recognition and management. CG90. London: NICE, 2009.

5 Davies J, Read J. A systematic review into the incidence, severity and duration of antidepressant withdrawal effects: are guidelines evidence-based? Addict Behav 2019;97:111-21.

6 Huijbers MJ, Spinhoven P, Spijker J, et al. Discontinuation of antidepressant medication after mindfulness-based cognitive therapy 
for recurrent depression: randomised controlled non-inferiority trial. Br J Psychiatry 2016;208:366-73.

7 Maund E, Stuart B, Moore M, et al. Managing antidepressant discontinuation: a systematic review. Ann Fam Med 2019;17:52-60.

8 Bowers HM, Kendrick T, Glowacka M, et al. Supporting antidepressant discontinuation: the development and optimisation of a digital intervention for patients in UK primary care using a theory, evidence and person-based approach. BMJ Open 2020;10:e032312.

9 Segal WJMG, Teasdale JD. Mindfulness-Based cognitive therapy for depression. 2nd ed. Guilford Press, 2012.

10 Kuyken W, Warren FC, Taylor RS, et al. Efficacy of MindfulnessBased cognitive therapy in prevention of depressive relapse: an individual patient data meta-analysis from randomized trials. JAMA Psychiatry 2016;73:565-74.

11 Tickell A, Byng R, Crane C, et al. Recovery from recurrent depression with mindfulness-based cognitive therapy and antidepressants: a qualitative study with illustrative case studies. BMJ Open 2020;10:e033892.

12 Kuyken W, Byford S, Taylor RS, et al. Mindfulness-based cognitive therapy to prevent relapse in recurrent depression. J Consult Clin Psychol 2008;76:966-78.

13 Kuyken W, Hayes R, Barrett B, et al. Effectiveness and costeffectiveness of mindfulness-based cognitive therapy compared with maintenance antidepressant treatment in the prevention of depressive relapse or recurrence (prevent): a randomised controlled trial. Lancet 2015;386:63-73.

14 Bockting CLH, Klein NS, Elgersma HJ, et al. Effectiveness of preventive cognitive therapy while tapering antidepressants versus maintenance antidepressant treatment versus their combination in prevention of depressive relapse or recurrence (DRD study): a threegroup, multicentre, randomised controlled trial. Lancet Psychiatry 2018;5:401-10.

15 Eveleigh R, Muskens E, Lucassen P, et al. Withdrawal of unnecessary antidepressant medication: a randomised controlled trial in primary care. BJGP Open 2018;1:bjgpopen17X101265

16 Wentink C, Huijbers MJ, Lucassen P, et al. Discontinuation of antidepressant medication in primary care supported by monitoring plus mindfulness-based cognitive therapy versus monitoring alone: design and protocol of a cluster randomized controlled trial. BMC Fam Pract 2019;20:105.

17 O'Brien BC, Harris IB, Beckman TJ, et al. Standards for reporting qualitative research: a synthesis of recommendations. Acad Med 2014;89:1245-51.

18 Huijbers MJ, Spijker J, Donders ART, et al. Preventing relapse in recurrent depression using mindfulness-based cognitive therapy, antidepressant medication or the combination: trial design and protocol of the moment study. BMC Psychiatry 2012;12:125.

19 First MB, Gibbon M, Spitzer RL, et al. User's guide for the structured clinical interview for DSM-IV axis I Disorders - Research version. New York: Biometrics Research Department, New York State Psychiatric Institute, 1996.

20 Rush AJ, Gullion CM, Basco MR, et al. The inventory of depressive symptomatology (IDS): psychometric properties. Psychol Med 1996;26:477-86.

21 Hardeveld F, Spijker J, De Graaf R, et al. Prevalence and predictors of recurrence of major depressive disorder in the adult population. Acta Psychiatr Scand 2010;122:184-91.

22 Boeije H. A purposeful approach to the constant comparative method in the analysis of qualitative interviews. Quality and Quantity 2002;36:391-409.

23 Scholten WD, Batelaan NM, van Oppen P, et al. The efficacy of a group CBT relapse prevention program for Remitted anxiety disorder patients who Discontinue antidepressant medication: a randomized controlled trial. Psychother Psychosom 2018;87:240-2.

24 Maund E, Dewar-Haggart R, Williams S, et al. Barriers and facilitators to discontinuing antidepressant use: a systematic review and thematic synthesis. J Affect Disord 2019;245:38-62.

25 Meyer JH, Wilson AA, Sagrati S, et al. Serotonin transporter occupancy of five selective serotonin reuptake inhibitors at different doses: an [11C]DASB positron emission tomography study. Am J Psychiatry 2004;161:826-35.

26 Horowitz MA, Taylor D. Tapering of SSRI treatment to mitigate withdrawal symptoms. Lancet Psychiatry 2019;6:538-46.

27 Groot PC, van Os J. Antidepressant tapering strips to help people come off medication more safely. Psychosis 2018;10:142-5.

28 Bowers HM, Williams SJ, Geraghty AWA, et al. Helping people discontinue long-term antidepressants: views of health professionals in UK primary care. BMJ Open 2019;9:e027837.

29 Chouinard G, Chouinard V-A. New classification of selective serotonin reuptake inhibitor withdrawal. Psychother Psychosom 2015;84:63-71.

30 Wentink C, Huijbers MJ, Lucassen PL, et al. Enhancing shared decision making about discontinuation of antidepressant medication: a concept-mapping study in primary and secondary mental health care. Br J Gen Pract 2019;69:e777-85.

31 Baldessarini RJ, Tondo L, Ghiani C, et al. Illness risk following rapid versus gradual discontinuation of antidepressants. Am J Psychiatry 2010;167:934-41. 